\title{
Effect of self-assembly aggregation on physical properties of non- aqueous ionogels based on LMWG
}

\section{Bielejewski iD ${ }^{1}$}

Received: 6 June 2018 / Accepted: 22 September 2018 / Published online: 3 October 2018

(c) The Author(s) 2018

\begin{abstract}
In recent years the low-molecular-weight gelators (LMWG) started to be used as a hardener for liquid electrolytes to create ionogels (OIG). The characteristic properties of gels created by LMWG, like thermal reversibility, very low critical concentration of the gelator, and huge versatility of possible compounds that can be used, were thought to give them advantage over commercial polymer gel electrolytes (PGE). However, the PGE are still dominating, mainly thanks to its superior mechanical resistance and wide temperature range when compared to OIG. The narrow temperature range of the gel phase existence in case of OIGs is the biggest disadvantage. This paper reports the interdisciplinary study on physical properties of created ionogels with nonaqueous electrolyte solutions of quaternary ammonium salts (TAMBr, TEABr, $\mathrm{TBABr}$ ) and low-molecular-weight gelators for different molar concentrations of the electrolyte. It will be shown how the self-assembly aggregation phenomenon can be used to extend the temperature range of the ionogel phase existence. The thermal scanning conductometry (TSC) method was used to investigate the electric properties of the ionogels. To investigate the transport properties of the cations and solvent molecules in the gel and sol phase, the NMR diffusometry method was used. For identification of intermolecular interaction in studied systems, the NMR spectroscopy method was used and to study how the different salts influence on the properties of used solvent, the tuning-fork vibration viscosimetry method was used.
\end{abstract}

\section{Graphical Abstract}

An enhanced thermal stability effect in renewable organic ionic gels (OIGs) achieved by controlling the self-assembly process during gelation stage.

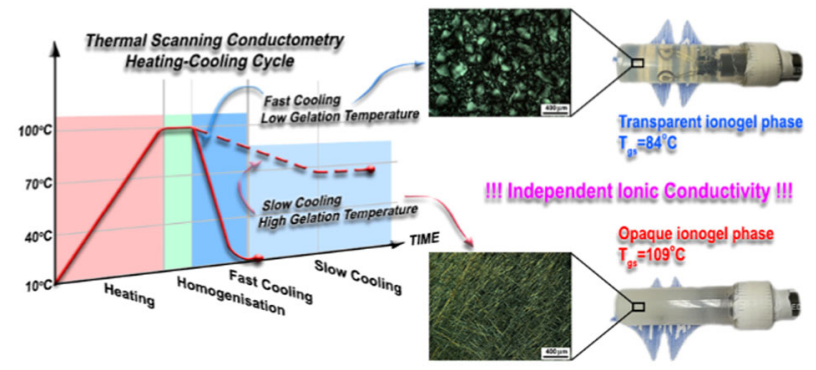

Electronic supplementary material The online version of this article (https://doi.org/10.1007/s10971-018-4831-7) contains supplementary material, which is available to authorized users.

M. Bielejewski

bielejewski@ifmpan.poznan.pl

1 Institute of Molecular Physics Polish Academy of Sciences, ul. M. Smoluchowskiego 17, 60-179, Poznan, Poland 


\section{Highlights}

- The thermal scanning conductometry (TSC) method was used to provide more details in investigations of thermally reversible ionic gels with respect to classical conductometry. Based on obtained data, the enhancement of thermal stability was detected.

- Performed studies showed that the microstructure of the 3D gel matrix created by self-assembly process based on hydrogen bonding is responsible for thermal properties of the ionogel phase.

- The detailed NMR diffusometry studies showed that translational motion of the investigated cations has a mobility comparable but lower in the gel phase with respect to liquid electrolyte. On the basis of obtained data it was concluded that 3D gel matrix does not compose any geometrical restrictions for ions in the gel phase.

- In this paper it has been shown how the tuning-fork vibration viscometry measurements can deliver information about stability of created electrolyte solutions.

- Appropriate thermal processing (possible to achieve with TSC method) during gelation stage determines the selfassembly process leading to formation of different gel matrices with enhanced thermal properties or different optical appearance of the ionogel phase (transparent or opaque).

Keywords Ionic gels $\cdot$ Thermal scanning conductometry $(\mathrm{TSC}) \cdot$ Diffusion NMR $\cdot$ Self-assembly aggregation

\section{Introduction}

In recent years, the use of low-molecular-weight gelators to create ionogels gave hope for obtaining new materials for energy storage, transmission, and conversion that can be used in electronic devices. The liquid electrolytes, which are commonly used in various applications, are known sometimes to cause leakage problems, so they need to be encapsulated. Therefore searching for mechanically rigid quasi-solid or solid alternative is desirable. The commercial polymer gel electrolytes solve many problems, however, introduce some shortcomings that need to be solved in the near future. Some of the main disadvantages of PGE are slowly deteriorating internal structure, what limits the lifetime of the device, low conductivity, complicated manufacturing and necessity for long-term storage of used or damaged devices on a garbage dump. Looking for a cause of these limitations, one has to mention the mechanism creating the polymer gel electrolyte phase, which is chemical polymerization reaction. To solve the mentioned problems, one has to think about a different way of creating the gel phase. An alternative solution is to use non-covalent interactions between the molecules of the gelator, rather than the covalent ones. This approach leads to physical gelation and thermally reversible systems which can be renewed every time it is needed to restore the initial properties. In the current literature, there are many examples of low-molecular-weight gelators that can be used to create different physical gel phases. Many of them can be used to create thermally reversible ionogels in some cases with temperature range around $100^{\circ} \mathrm{C}[1-7]$. Another advantage of the LMWG is its natural origin what makes them neutral to the environment and biodegradable. The mechanism of the gelation in case of physical gels is hierarchically organized self-assembly process macroscopically expressed in the creation of 3D matrix composed of gelator molecules based on non-covalent interactions. As the final thermal stability and temperature range of the gel phase is dependent on the rigid skeleton made of gelator molecules which self-support the liquid phase, therefore making it stronger should result in extending the phase transition temperature. The driving forces responsible for gelation could be the electrostatic interaction, van der Waals interaction, $\pi-\pi$ stacking, coordination, London forces, but mostly hydrogen bonds [8]. Depending on the chemical structure of the gelator it is possible that more than one kind of interactions are active and take part in gelation. Therefore, controlling and influencing on them can lead to a change of the matrix and improvement of the structural parameters like size and shape of the aggregates, to form a stronger and durable gel matrix. Fortunately, these interactions are dependent on the external conditions, what can be used to control the gelation process by setting appropriate gelation temperature and temperature change rate. In this paper, we have studied the influence of mentioned parameters on the type of the molecular aggregates creating the gel matrix, no other modification into the structure of the gelator or electrolyte solution was made. The growing significance of the physical gels can be observed on the basis of their practical applications in a variety of different fields of industry (in pharmaceutics, medicine, enginery, electronics, etc.) [9-17]. In case of the ionogels, the weakest point is low phase transition temperature (even much below $100{ }^{\circ} \mathrm{C}$ ) what makes them lose with PGE, even if using polymers have some drawbacks (e.g., disposal of used elements). The high mechanical strength and wide temperature range of existence in case of polymer gel electrolytes make them desirable product already present on the market [18, 19]. Therefore, if OIGs are going to be an alternative for PGEs, its temperature range of the gel phase existence have to be 
extended. This paper will demonstrate how the change of thermal parameters during the gelation process influence on the self-assembly mechanism, leading to extending the temperature range of the gel phase existence. In the studied system, we have achieved an enhancement of $10-25^{\circ}$, depending on the composition, approaching and going beyond the $100{ }^{\circ} \mathrm{C}$ of the gel to sol $T_{\mathrm{gs}}$ phase transition temperature.

\section{Experimental section}

\subsection{Materials and preparation procedures}

Methyl-4,6- $O$-(p-nitrobenzylidene)- $\alpha$-D-glucopyranoside (1) was used as a low-molecular-weight gelator (LMWG) to prepare the ionogels with a nonaqueous solution of tetramethylammonium, tetraethylammonium, and tetrabutylammonium bromide in a wide range of the salt molar concentration. The chemical synthesis of $\mathbf{1}$ was described elsewhere [20].

The electrolyte solutions were prepared by mixing appropriate amounts of the TMABr, TEABr, and $\mathrm{TBABr}$ salts with glycerol to obtain solutions at a molar concentration of the salt in the range from 0.5 to $2.0 \mathrm{M}$. During preparation process the mixtures were heated and stirred to obtain complete dissolution of the solute, followed by cooling at ambient temperature. Next, such prepared electrolytes were investigated for stability by viscosity and TSC measurements. Afterwards were used as reference samples and to prepare ionogels with $\mathbf{1}$.

The ionogels were prepared by dissolving the gelator $\mathbf{1}$ in the electrolyte solution of given molar concentration. During this process the mixture was heated and stirred in a flame-sealed glass tube. The gelation process takes place during cooling down of the mixture, which at this point turns from sol to gel phase. To obtain transparent, thermally reversible and stable gels the cooling rate has to be fast. Moreover, due to the nature of physical gelation, it is crucial to remain exactly the same cooling conditions for all samples, as the properties of obtained gel phases depend on the thermal treating. To ensure the reproducibility of ionogel samples, we used TSC protocol to perform the cooling at given external conditions. To investigate the influence of cooling procedure on final properties of created ionogels, the gelation temperatures were set to 10 or $60^{\circ} \mathrm{C}$ achieved with 7 or $2{ }^{\circ} \mathrm{C} / \mathrm{min}$ cooling rates.

\subsection{Thermal scanning conductometry (TSC) measurements}

The ionic conductivity of electrolyte solutions and ionogels was investigated using a thermal scanning conductometry
(TSC). This unique method allows to follow dynamical changes of the samples' state during heating and cooling stages, what enables us to observe how the properties of the ionogel change when coming to the sol phase and back to the gel phase. To measure conductivity the Mettler-Toledo S230 SevenCompact digital conductivity meter equipped in InLab 710 four-electrode conductivity cell was used. The cell constant was calibrated using $1.413 \mathrm{mS} / \mathrm{cm}$ standard (aqueous solution of $\mathrm{KCl} 0.01 \mathrm{M}$ ). The investigated samples were put to polypropylene vials closed by screw caps with a rubber ring. The vials can be heated up to $120^{\circ} \mathrm{C}$, the tight and firm closing allows to avoid evaporation of water at high temperatures and the inner diameter of the vials match the outer diameter of the InLab 710 sensor. All measurements were performed with the use of the home-build temperature controller allowing to change the temperature of the sample linearly on heating and cooling with different temperature change rates. The sample in polypropylene vials with InLab 710 sensor was placed in a chamber which controls the samples' temperature. The heating/cooling medium was nitrogen gas flowing through the temperature chamber. The initial temperature of the nitrogen gas was lowered down to $263.15 \mathrm{~K}$ with the aid of AirJet XR refrigerator. The TSC measurements were carried out in heating-cooling cycles with a set temperature change rates $\left(0.5^{\circ} \mathrm{C} / \mathrm{min}\right.$ during heating and $\sim 7$ or $2{ }^{\circ} \mathrm{C} / \mathrm{min}$ during cooling). The basics of the TSC method are given elsewhere [21].

\subsection{NMR spectroscopy and diffusometry measurements}

The NMR diffusion measurements were performed on a Bruker Avance III HD spectrometer operating at $500 \mathrm{MHz}$ of ${ }^{1} \mathrm{H}$ Larmor frequency and coupled to $11.4 \mathrm{~T}$ superconducting magnet. The system is equipped with gradient unit and diffusion probe with the coil of 5-mm diameter allowing to perform pulse field gradient (PFG) ${ }^{1} \mathrm{H}$ NMR diffusion experiments using maximal gradient strength in $z$ direction of $30 \mathrm{~T} / \mathrm{m}$. The measurements were performed as a function of temperature from 293.15 to $353.15 \mathrm{~K}$, with the use of a pulse gradient stimulated echo (PGSTE) sequence for both electrolyte solution and ionogel samples. The recorded signal is attenuated due to molecules' motion taking place during so-called diffusion time $\Delta$ leading to phase encoded echo signals. The relation between observed signal attenuation and experimental parameters is given by the following equation:

$\frac{A(g)}{A(0)}=\exp \left[-\gamma^{2} \delta^{2} g^{2} D\left(\Delta-\frac{\delta}{3}\right)\right]$

where $A(g)$ and $A(0)$ are echo signal intensities, respectively with and without magnetic field gradient pulse applied, $\gamma$ is 
the gyromagnetic ratio and $D$ is the self-diffusion coefficient. The measurements were conducted as a function of the magnetic field gradient strength $g$, which was varied in 32 steps from 0 to $2 \mathrm{~T} / \mathrm{m}$. The parameters $\delta$ and $\Delta$ were equal to 2 and $75 \mathrm{~ms}$, respectively, and were kept constant for all experiments. The diffusion coefficient $D$ can be directly related to the mean-square displacement $\left\langle z^{2}\right\rangle$ observed in the direction of applied PFG, by the equation:

$z^{2}=2 D t$

where $t=\Delta-\delta / 3$, is equal to so-called diffusion time $\Delta$.

Because of very low concentration of the gelator molecules and rigidity of the gel matrix, the measured signals originate only from residual water and cations protons from the used salts.

In first approximation concerning the cation size and shape, the measured diffusion coefficient $D$ can be related to the viscosity using Stokes-Einstein relation in the following form:

$D=\frac{k_{B} T}{6 \pi \eta r}$

where $k_{B}$ is the Boltzmann constant, $T$ is the temperature, $\eta$ is the viscosity coefficient, and $r$ is the radius of the spherical approximation of the cation.

\subsection{Vibrational viscometry measurements}

The static viscosity was measured with tuning-fork vibration viscometer SV10 from A\&D Weighting, Japan. The temperature was regulated by home-built variable temperature controller and changed linearly in cooling and heating cycles. The sample volume of $10 \mathrm{ml}$ was used for all measurements and the viscometer was calibrated using Milli-Q distilled water and S600 viscosity standard from Paragon Scientific Ltd. The basics of the tuning-for vibrational viscometry method are given elsewhere [22].

\section{Results and discussion}

\subsection{Chemical composition and physical stability of investigated electrolyte solutions for OIGs}

One of the limiting factors which, from definition, influence on the usable temperature range is the boiling point of the solvent used to prepare the electrolyte solution. Therefore, in current research we have used a glycerol to prepare all electrolytes, because its boiling temperature is around $160^{\circ}$ $\mathrm{C}$ and glycerol it-self is harmless to human organism. As source of the charge carriers we have chosen quaternary ammonium salts as they are known from good solubility

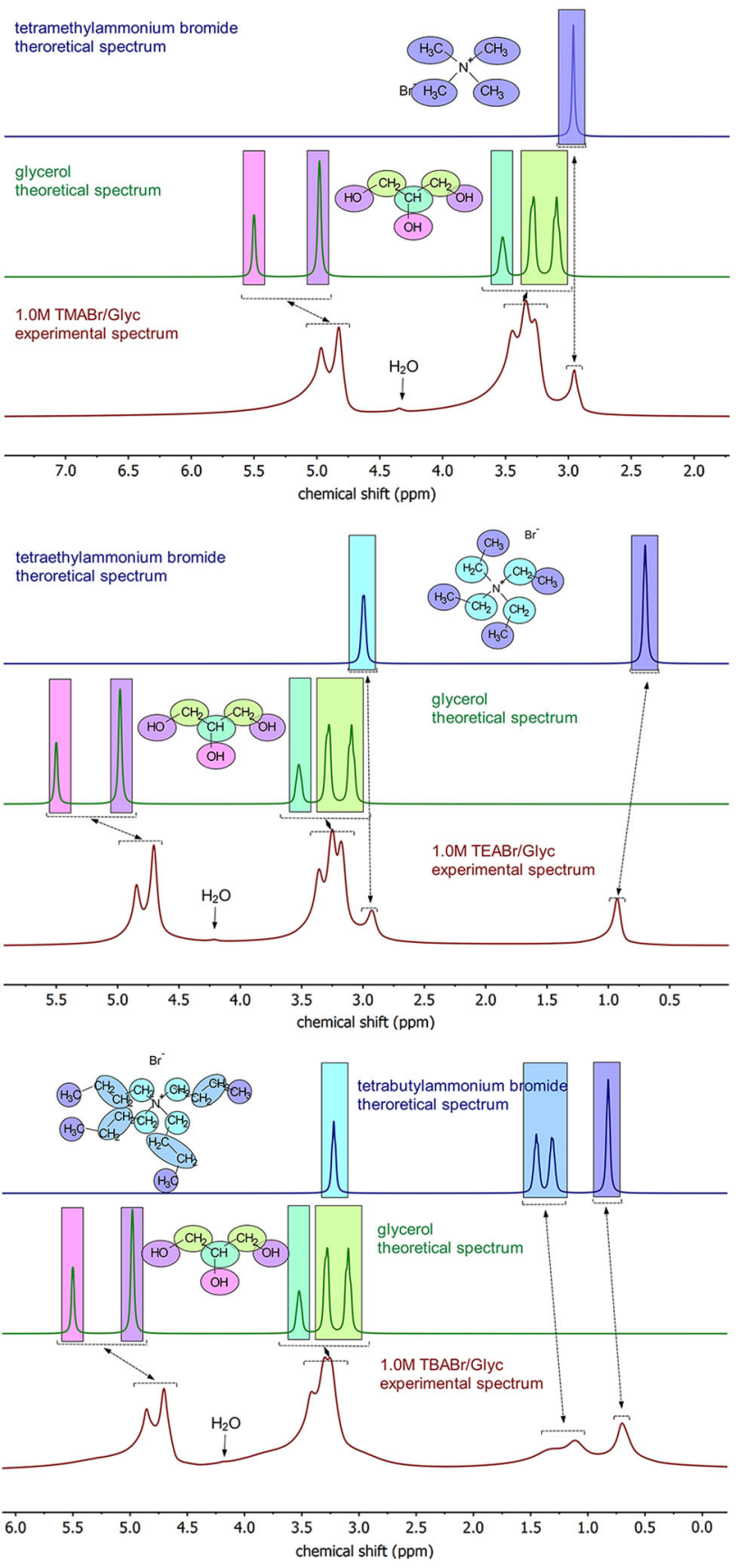

Fig. 1 The experimental ${ }^{1} \mathrm{H}$ NMR spectra of TMABr, TEABr, and $\mathrm{TBABr}$ electrolyte solutions together with its theoretical simulations used for line assignments

what allowed us to obtain high concentrations of ions in our electrolyte solutions. The chosen salts were: tetramethylammonium bromide ( $\mathrm{TMABr}$ ), tetraethylammonium bromide (TEABr), and tetrabutylammonium bromide (TBABr), prepared in concentrations from 0.5 to $2.0 \mathrm{M}$. Figure 1 shows the experimental ${ }^{1} \mathrm{H}$ NMR spectra obtained for all three salts at $1.0 \mathrm{M}$ concentration and $20^{\circ} \mathrm{C}$, together with theoretical ones used for line assignments. As can be seen the NMR lines are recognizable and quite well 
separated what will be important in further analysis of the self-diffusion coefficients for both liquid electrolyte and ionogel phases. To check whether the created electrolytes are chemically stable, all samples were investigated in function of temperature from 20 to $80^{\circ} \mathrm{C}$, which is the highest possible to achieve in our NMR setup. The temperature dependence of the NMR spectra of investigated electrolyte solutions are presented on Fig. S1 in supporting information. On their basis, we can conclude that ions of used salts are stable in glycerol. To verify if prepared solutions are stable in time, we have investigated the time and temperature dependence of the static viscosity. In case of any change in the electrolyte solution state, e.g., resulting from precipitation process the viscosity of sample should change. Figure 2 shows the temperature dependencies of the static viscosity recorded for all three salts and three molar concentrations of the ions. As can be seen in all cases except one, we can observe nice dependencies without any disturbances. Figure S2 in supporting information shows the viscosity of a electrolyte solution at given concentration in function of the ion type. In case of $2.0 \mathrm{M}$ concentration of $\mathrm{TMABr} / \mathrm{Glyc}$ electrolyte solution a precipitation occurred. To investigate this case in more details, we have measured the static viscosity of $1.0 \mathrm{M} \mathrm{TMABr} / \mathrm{Glyc}$ electrolyte solution in function of time, to check when the precipitation process starts. Figure 3 shows how the system under investigation evolve in time. At the beginning, the electrolyte solution was heated to $100^{\circ} \mathrm{C}$ to ensure total dissolution of the salt, next the temperature was stabilized at $23^{\circ} \mathrm{C}$ and the viscosity was measured over time.

As can be seen almost immediately after reaching $23^{\circ} \mathrm{C}$, the viscosity was gradually increasing, although macroscopically no change was observed. This increase of static viscosity is the evidence for slow precipitation process taking place in the system. At the end of measurements, the precipitated salt was seen with naked eye. This behavior will be confirmed by TSC measurements, presented in following sections. On the basis of the viscosity measurements, we have found that only electrolyte solution with TEABr will give the best results in ionogel phase. The electrolyte solution with $\mathrm{TMABr}$ is precipitating from the solution and the electrolyte solution with $\mathrm{TBABr}$ shows increase of the viscosity what would apply directly on conductivity of created OIG.

In case of the TEABr salt, we observe decrease of the static viscosity with increase of the ion concentration. This effect can be understood if we take into account that glycerol molecules forms effectively strong hydrogen bonds between themselves. The presence of the charged species in form of dissociated salt ions disturb this hydrogen bond network what leads to smaller glycerol aggregates and in result decrease the static viscosity of the electrolyte

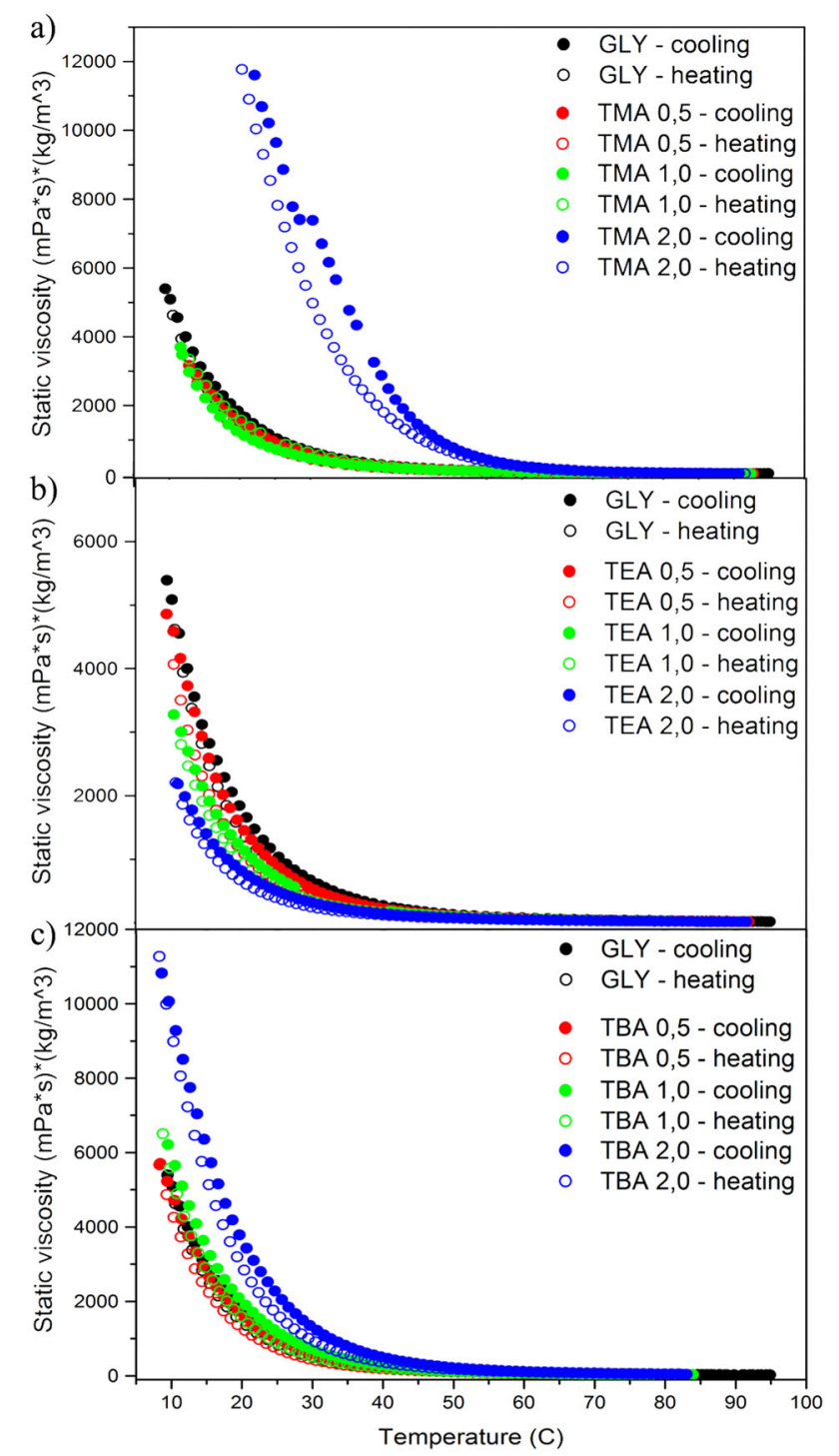

Fig. 2 The temperature dependence of the static viscosity recorded for all investigated electrolyte solutions at $0.5,1.0$, and $2.0 \mathrm{M}$ concentrations (GLY glycerol, TMA tetramethylammonium bromide a, TEA tetraethylammonium bromide b, TBA tetrabuthylammonium bromide c)

solution. The effect of tailoring the glycerol aggregates and increasing the dynamics was also observed on the temperature dependence of the TEABr/Glyc ${ }^{1} \mathrm{H}$ NMR spectra (Fig. 5), where the signals from hydroxyl groups with increase of temperature are averaging and approach themselves to create one line at the highest temperature. Whereas for pure glycerol, where the hydrogen bond network between glycerol molecules is strong and developed, we can see two lines from the hydroxyl groups up to the highest temperatures (Fig. 4). In rest of the studies only the electrolyte solution with TEABr and ionogel with it will be discussed. As a reference system a pure glycerol (as solution) and physical gel with it will be used. 


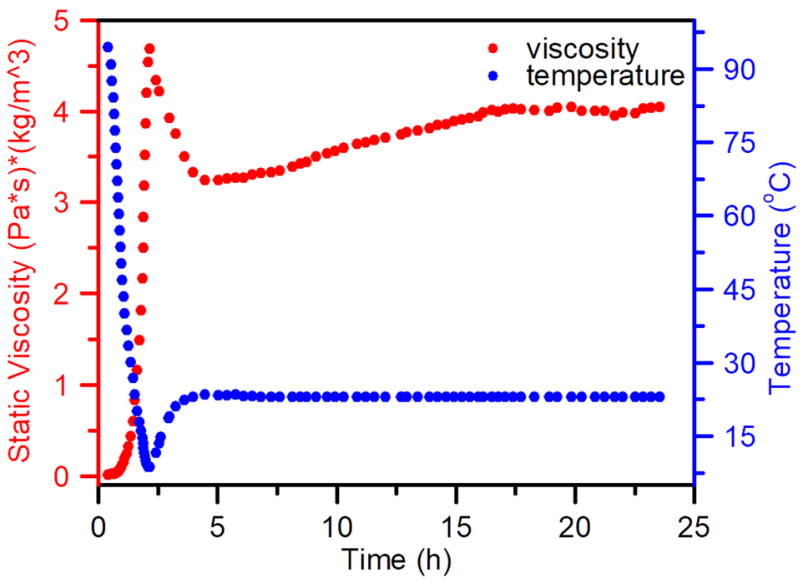

Fig. 3 The time dependence of the static viscosity recorded for $1.0 \mathrm{M}$ TEABr/Glyc electrolyte solution
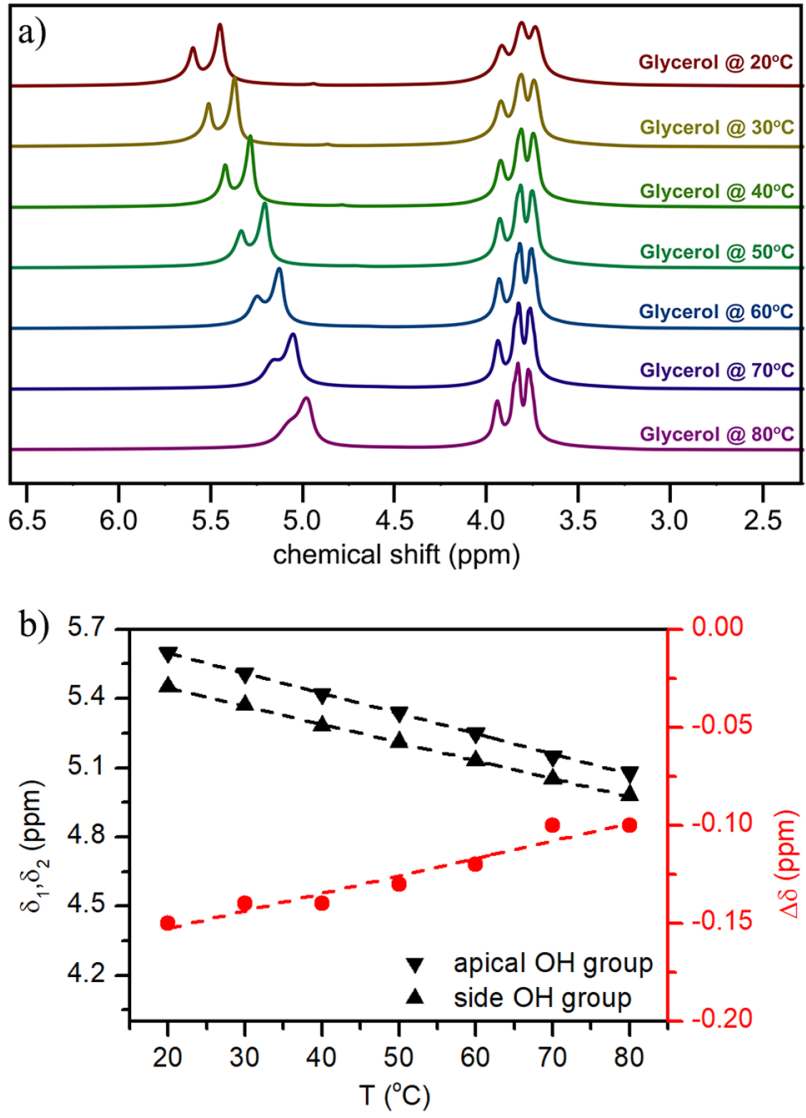

Fig. 4 The temperature dependence of ${ }^{1} \mathrm{H}$ NMR spectrum of glycerol a, together with analysis of the hydroxyl groups chemical shift $\mathbf{b}$

\subsection{Molecular interactions and dynamics investigated by NMR spectroscopy and diffusometry method}

The intermolecular interactions occurring between molecules of the electrolyte solutions and ionogel phases were investigated with NMR spectroscopy method. The measurements were performed on protons $\left({ }^{1} \mathrm{H}\right)$ therefore, observed signals in the spectra correspond to glycerol molecules and $\mathrm{TEA}^{+}$cations only. Also a trace of water molecules has been detected, as glycerol is hygroscopic. The anion, which for all salts is the same $\left(\mathrm{Br}^{-}\right)$, is not detectable under our experimental conditions. All measurements were conducted for three molar concentrations $(0.5,1.0$, and $2.0 \mathrm{M})$ as a function of temperature. Additionally a reference sample with pure glycerol was also measured. For better clearance, only data for $1.0 \mathrm{M}$ concentration of the electrolytes will be presented as representative results. Figure 4 presents the spectra recorded for pure glycerol as a function of temperature (for assignments of the lines please refer Fig. 1). As can be seen, only the lines coming from hydroxyl groups are displaying change of the chemical shifts. This can be understood as those groups in glycerol are taking part in hydrogen bonding, which are sensitive to temperature change. Figure $4 \mathrm{~b}$ shows the relative difference in chemical shifts $\Delta \delta$ between side and apical hydroxyl group. The linear dependence of $\Delta \delta$ observed for pure glycerol in liquid state was also observed for all electrolyte solutions at liquid state. Figure 5 shows the temperature dependence of the NMR spectrum recorded for $1.0 \mathrm{M}$ concentration of TEABr/Glyc electrolyte solution (for assignments of the lines please refer Fig. 1).

As can be seen, lines from both components (glycerol and TEABr) are well separated. The addition of salt did not have an influence on the starting $\Delta \delta$ between hydroxyl groups, but increases the chemical shift of the apical hydroxyl group at higher temperatures. This effect can be understood if we take into account the presence of dissociated ions of the salt. In the studied case, the cation TEA ${ }^{+}$has protons that can also take part in hydrogen bonding; moreover, both ions introduce an electrostatic potential which also has an influence on a created network of hydrogen bonds between glycerol molecules. This conclusion remains in agreement with the results from viscosity measurements, where we observed a decrease of the static viscosity upon addition of TEABr salt. The decreased viscosity would imply that molecules are more free to move, this can be understood if we assume that the presence of the salt ions disturbs the network of hydrogen bonds between glycerol molecules leading to smaller aggregates of solvent molecules. The reduced size of glycerol aggregates induces an increase of freedom of what is implied for higher dynamics with an increase of temperature, making the signals from hydroxyl groups more averaged in what can be observed as corresponding lines coming closer to each other in the NMR spectra. In the studied case, the difference in chemical shifts of the hydroxyl groups in TEABr/Glyc electrolyte is twice as small as in pure glycerol at $80^{\circ} \mathrm{C}$. In Fig. 6, the recorded NMR spectra for a molecular gel with 

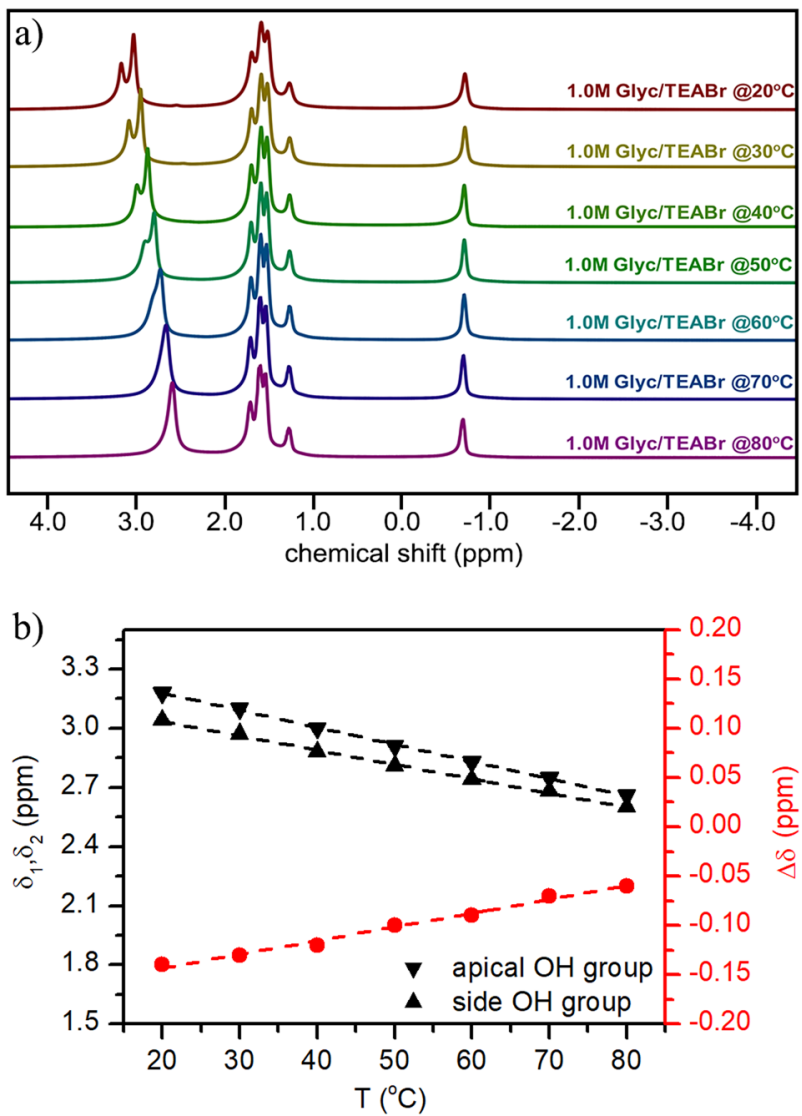

Fig. 5 The temperature dependence of ${ }^{1} \mathrm{H}$ NMR spectrum of $1.0 \mathrm{M}$ $\mathrm{TEABr} /$ glycerol electrolyte solution $\mathbf{a}$, together with analysis of the hydroxyl groups chemical shift $\mathbf{b}$

glycerol (reference system) as a function of temperature are presented.

As can be seen in the gel sample, we observe similar behavior with one difference which is visible at low temperatures. In the temperature range from 20 to $40{ }^{\circ} \mathrm{C}$, the $\Delta \delta$ between hydroxyl groups is kept constant. This was not observed in liquid state, therefore, this effect has to be connected with the existence of the gel matrix which gives the whole system additional rigidity and transforms it to gel phase. From Fig. 6a, we see that all three hydroxyl groups are changing its chemical shifts with temperature, however at the beginning in the same way. This can be explained by assuming that the hydrogen bonds in which they are involved are affected in the same way by temperature. Such behavior would mean that the gel phase is stable and all interactions are kept in balance, what is possible if we assume that the extent of energy that we deliver to sample is taken over by the gel matrix. When we cross certain energy (temperature) which cannot be handled by the matrix, we observe that the chemical shifts start to behave as in the liquid state. This means that we might be approaching the temperature when the phase transition process starts. However, it needs to be kept in mind that such gel matrices
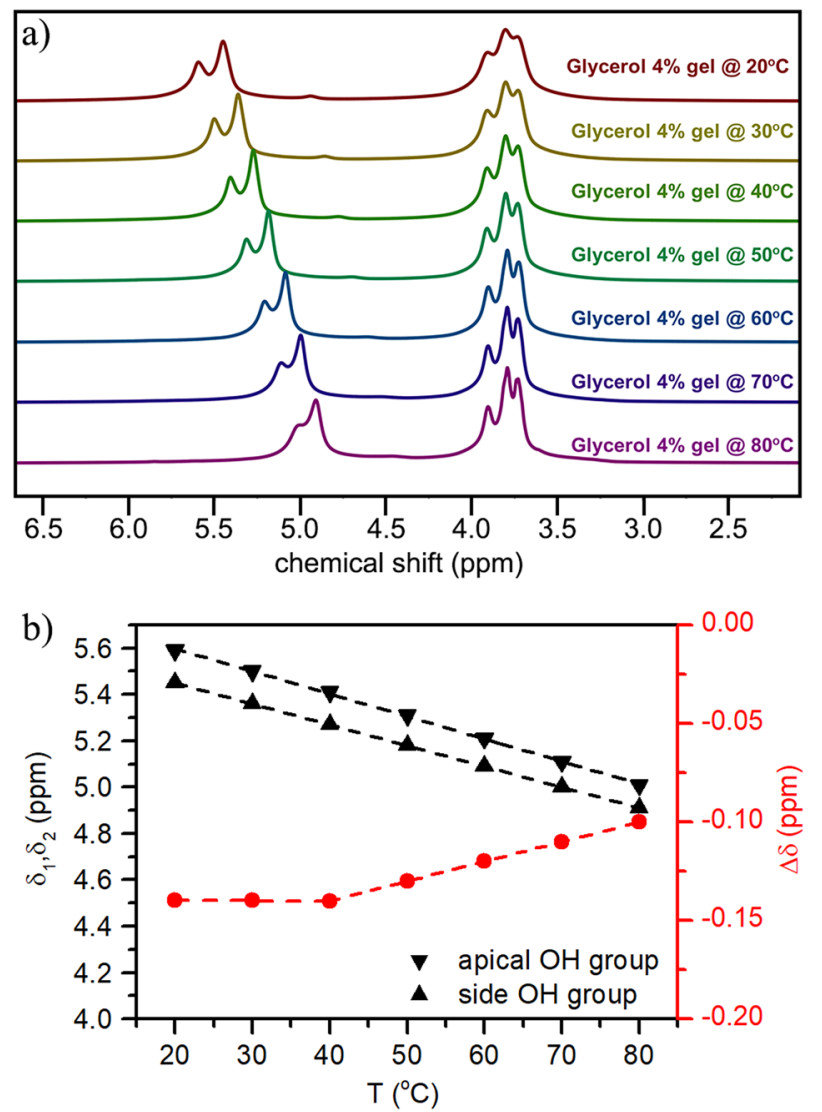

Fig. 6 The temperature dependence of ${ }^{1} \mathrm{H}$ NMR spectrum of a molecular gel made with glycerol - a reference system a, together with analysis of the hydroxyl group chemical shift $\mathbf{b}$

are inhomogeneous; therefore, the phase transition is not a sharp one and can start before we will see a liquid in a sample. This also proves how sensitive is the NMR technique. Figure 7 presents the same data but this time for an ionogel, and the analysis points to the same conclusions as for the reference system. The only difference is that the $\Delta \delta$ is kept constant to higher temperatures. This would mean that the ionogel system is more stable. We have observed such behavior earlier for other ionogel systems created by the same LMWG, of which the detailed studies were published elsewhere [23].

The translational dynamics of the solvent and ions was studied with a pulse gradient stimulated echo (PFGSTE) NMR method. The NMR diffusometry has already been proved to be a very precise tool in observation of diffusion behavior of small molecules of a liquid in physical gels [24-26].

The measurements were performed as a function of temperature for all investigated cases. Figure 8 shows an example of echo signal decay recorded at $20^{\circ} \mathrm{C}$ for TEABr/ Glyc electrolyte solution at $1.0 \mathrm{M}$ concentration. The data for the reference system as well as for the ionogel system are presented in supporting information in fig. S3 and fig. 

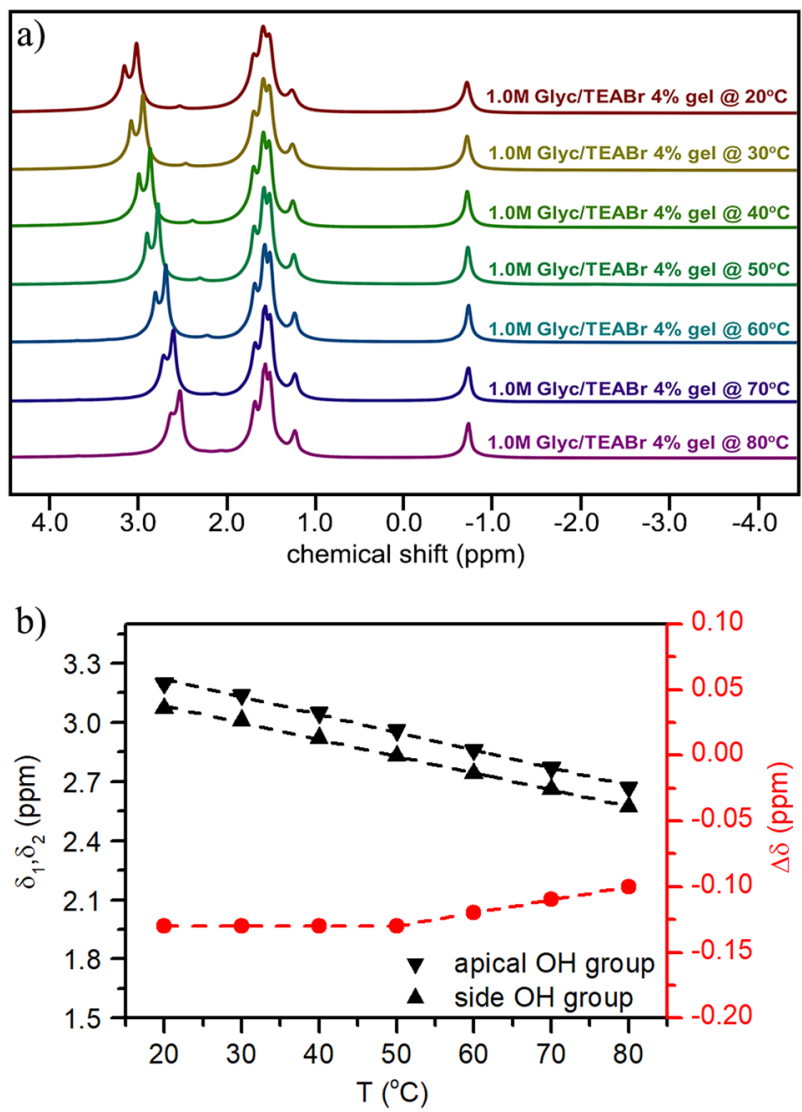

Fig. 7 The temperature dependence of ${ }^{1} \mathrm{H}$ NMR spectrum of an ionogel made with $1.0 \mathrm{M} \mathrm{TEABr} /$ glycerol electrolyte solution a, together with analysis of the hydroxyl group chemical shift $\mathbf{b}$

S4. As can be seen, all echo signal are well visible and separated, and solid lines in Fig. 8b represent the best fits of Eq. 1 to experimental points. For a reference system (with pure glycerol), we have observed a mono-exponential behavior for glycerol molecules and bi-exponential behavior for water signals. In all investigated cases of electrolyte solutions as well as for ionogels, we have seen a biexponential behavior of all lines except for the highest temperatures. Figure 9 shows the self-diffusion coefficient $D$ obtained by fitting Eq. 1 to experimental points for a reference system, $1.0 \mathrm{M}$ electrolyte solution, and ionogel system as a function of temperature.

The bi-exponential echo decay can be explained by the existence of two different diffusion coefficients for investigated molecules. The situation concerning translational motion in studied systems is rather complicated due to the effective hydrogen bonding process between glycerol and water and high probably also with cation molecules. This very dynamic hydrogen bonding pattern leads to a situation where we have the same molecules in different surroundings. In the simplest case for the reference system (Fig. 9a), we observe bi-exponential behavior only for water molecules. This can be easily understood if we keep in mind that
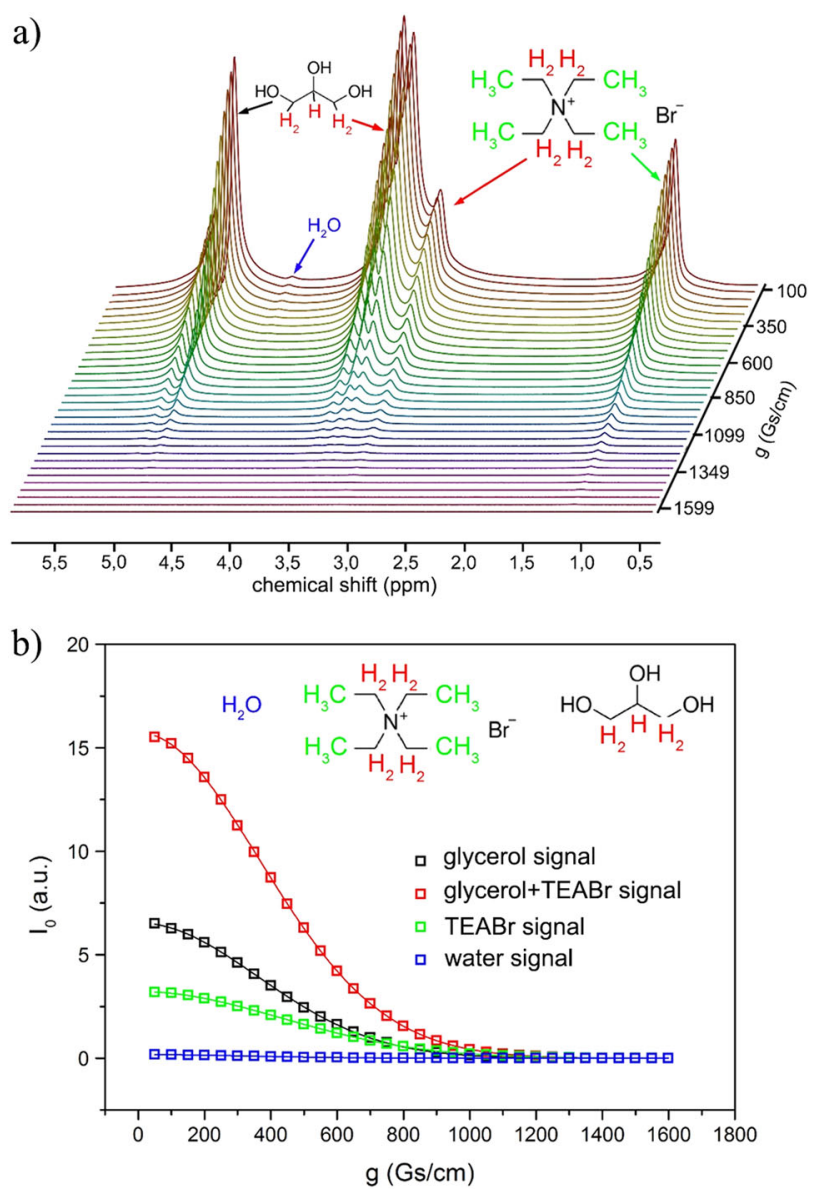

Fig. 8 The echo signal attenuation observed for 1.0 M TEABr/glycerol electrolyte solution at $20^{\circ} \mathrm{C}$ a, together with the best fit of Eq. 1 to experimental points $\mathbf{b}$

observed water comes from hygroscopic properties of glycerol; therefore, we can treat this situation as an analog to water-in-oil microemulsion, where water creates small droplets of liquid surrounded by glycerol. This situation is possible as the water content is very small in relation to glycerol. In such case, we have water which interacts directly with glycerol molecules and the one which is inside the droplet. The diffusion coefficient of water interacting with glycerol through hydrogen bonds is equal to the diffusion coefficient of glycerol molecules, whereas diffusion of water inside the droplets interacts only with other water molecules and has a higher diffusion coefficient. This situation is reflected by experimental data in Fig. 9a. In the case for an electrolyte, the situation becomes more complicated as we have additional components which bring in an electrostatic potential. Thus, the dissociated ions are surrounded by a solvation shell composed of solvent molecules. As a result, in the case of electrolyte solution (Fig. 9b) at low temperatures, we observe two diffusion coefficients for the cation. One comes from cations surrounded by glycerol and the second from cations 


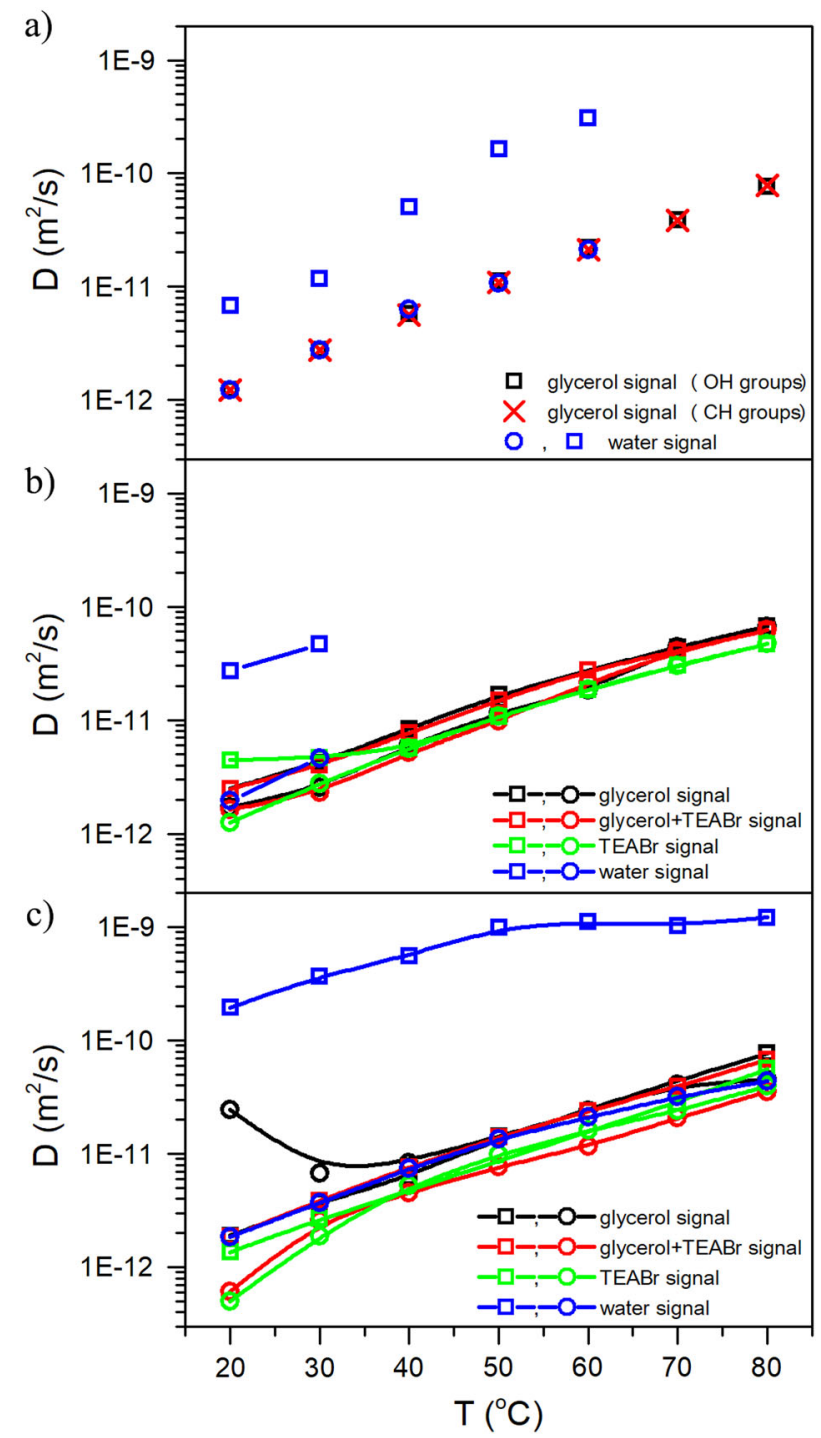

Fig. 9 The temperature dependence of the self-diffusion coefficient $D$ obtained from fitting the procedure for all components of glycerol (reference sample) a, 1.0 M TEABr/glycerol electrolyte solution $\mathbf{b}$, and ionogel with $1.0 \mathrm{M} \mathrm{TEABr} / \mathrm{glycerol}$ electrolyte solution c. The solid lines are only eye guides

surrounded by water. What is interesting is that the bifunctional behavior disappeared at higher temperatures and based on the value of the observed self-diffusion coefficient, we observed only cations surrounded by glycerol. Probably at higher temperatures, the water droplets are becoming too small for the cation to fit. The bi-functional behavior of glycerol is preserved to much higher temperatures, which means that we have glycerol in the solvation shell and outside it. At the highest temperatures, these two selfdiffusion coefficients average out. In the case of the ionogel system (Fig. 9c), the scenario is the same, only this time the bi-exponential behavior of glycerol is preserved up to the highest (possible to achieve in our setup) temperatures, meaning that the gel phase makes everything more rigid and stable. These results are in agreement with data obtained in NMR spectroscopy. As will be shown in the next section, the gel to sol phase transition temperature $T_{\mathrm{gs}}$ of a $1.0 \mathrm{M}$ TEABr/Glyc ionogel can be found in the range from above $80^{\circ}$ to above $90^{\circ} \mathrm{C}$. For this reason, it is completely understandable why we see the bi-exponential behavior for glycerol observed at $80^{\circ} \mathrm{C}$ in NMR diffusometry experiment, as the system is still in the ionogel phase.

By the use of Stokes-Einstein relation and the correlation between measured diffusion and viscosity coefficients, we have predicted that the system based on tetraethylammonium bromide is the one with the highest potential to achieve the highest ionic conductivity. As will be shown below, the $2.0 \mathrm{M}$ ionic gels with TEABr were characterized by the highest conductivity and stability in time. However, one has to keep in mind that there can be other limiting factors besides geometrical and motional restrictions, which can lead to a decrease or saturation of the observed conductivity. The ion-pairing effect is one of such processes, which was observed for $\mathrm{TBABr}$ but not in the case of TEABr.

\subsection{The ionic conductivity properties and gel to sol phase transition temperature of the ionogel phase}

The organic ionic gels (OIGs) are characterized with high ionic conductivity on the level of liquid electrolytes from which they are made of. The critical gelation concentration which is the minimum amount of the gelator molecules needed to create the ionogel phase can be as low as a couple of weight\%; thus, the solid matrix is only a very small addition to the liquid phase. On the contrary, the polymer gel electrolytes need much more of the gelator agent (polymer) which can constitute from 30 to $70 \%$ of the total weight. However, the most spectacular feature of the OIGs is their thermal reversibility which allows them to self-heal or reconstruct the internal structure to obtain nominal parameters after usage period or damage. As can be seen from the NMR diffusion experiments, the gel matrix does not introduce any obstacles or geometrical restrictions to mobility of the ions, when compared with liquid electrolytes. This conclusion is also confirmed by the TSC measurements, where the conductivity of an ionogel phase is on the same level as observed for liquid electrolyte. Moreover, in our other study concerning ionogels we observed the same behavior [27]. To determine which of the investigated electrolyte solutions of quaternary ammonium salts gives the best results, temperature dependences of the ionic conductivity for all salts at all electrolyte concentrations were measured and compared with themselves and each other. The obtained results are presented in supporting information in fig. S5 and fig. S6, and are in agreement with viscosity 


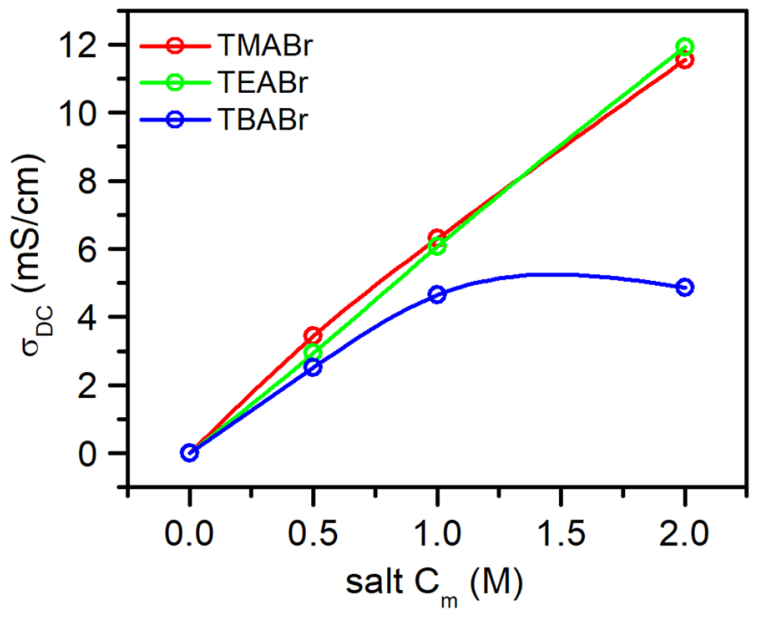

Fig. 10 The ionic conductivity of electrolytes made with $\mathrm{TMABr}$, $\mathrm{TEABr}$, and $\mathrm{TBABr}$ salts recorded at $80^{\circ} \mathrm{C}$

measurements pointing that the best performance was observed for TEABr salt. For a quick overview of the conductive properties of investigated electrolyte solutions in Fig. 10, the ionic conductivity of a given electrolyte solution at $80{ }^{\circ} \mathrm{C}$ as a function of molar concentration of the ions is presented.

To confirm that the TMABr salt precipitates from solution at $1.0 \mathrm{M}$ concentration, what was observed as a change of the viscosity measured at $23{ }^{\circ} \mathrm{C}$ as a function of time, the conductivity of that sample was measured in the same conditions. The obtained results are presented in supporting information in fig. S7 and are in agreement with viscosity data. Figure 11 presents the TSC curves recorded for TEABr/Glyc ionogel and reference data for a molecular gel with glycerol and the same LMWG. The TSC curves for the ionogels and the corresponding electrolyte solution at different molar concentrations are presented in fig. S8 in supporting information, showing that conductivity in the ionogel phase is on the same level as for liquid electrolyte for investigated concentration of ions.

As OIGs are physical gels, we have studied how the external conditions during the gelation stage will affect the final properties of the obtained ionogels. Thanks to the use of the TSC method, we could perform the full characteristics of ionic conductivity during the heating-cooling cycle and observe the self-assembly process which takes place and leads to creation of the 3D matrix of gelator molecules. We have programmed three different scenarios in which we changed the gelation temperature and cooling rate, influencing in this way on the kinetics of the self-assembly process which was based on hydrogen bonding. All experiments were measured independently three times giving the same results. Below, we discuss the ionogel sample created with $1.0 \mathrm{M} \mathrm{TEABr} / \mathrm{Glyc}$ electrolyte solution. In the first scenario, we used standard settings for the

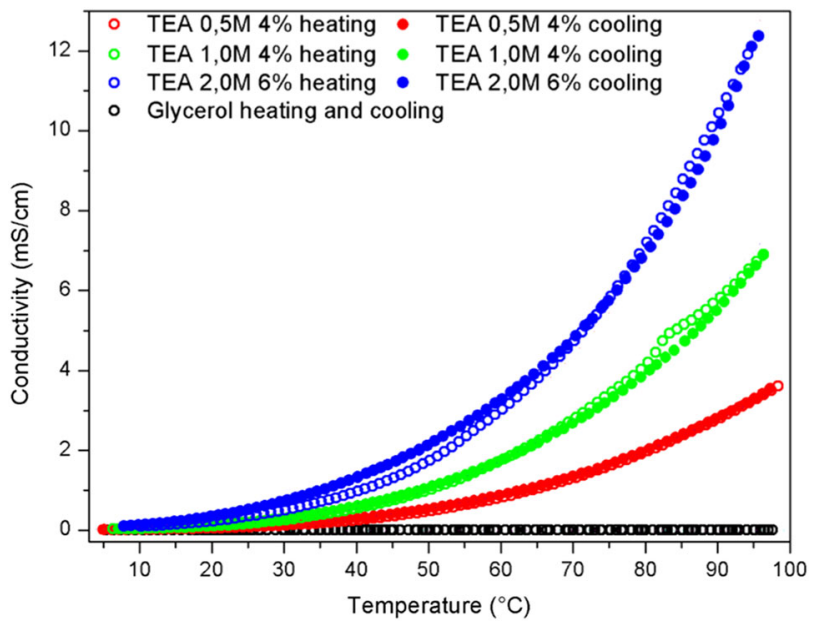

Fig. 11 The TSC curves recorded for ionogel with TEABr/glycerol electrolyte solutions at $0.5,1.0$, and $2.0 \mathrm{M}$ concentrations together with the reference system (molecular gel with glycerol)

cooling stage, which stands for fast cooling from the sol phase (gelation temperature $10^{\circ} \mathrm{C}$ and cooling rate of $7^{\circ} \mathrm{C} /$ min). As a result, we obtained a transparent ionogel phase. In the second scenario, we have decreased the cooling rate from 7 to $2{ }^{\circ} \mathrm{C} / \mathrm{min}$ but the gelation temperature was kept at $10^{\circ} \mathrm{C}$. As a result, we got an ionogel phase which was a mixture of a transparent and opaque gel phase. In the third scenario, we have kept the cooling rate at $2{ }^{\circ} \mathrm{C} / \mathrm{min}$ but we have changed the gelation temperature to $60^{\circ} \mathrm{C}$. As a result, we obtained a fully opaque ionogel phase. Figure 12 shows the TSC heating curves recorded after cooling from the sol phase in mentioned conditions.

As can be seen, no differences in conductivity of ionogels have been observed in the whole temperature range. Except for an anomaly which changes its position. The mentioned anomaly originates in phase transition from gel to sol phase and a start of bulk flow in the sample. The details of this analysis are explained in our previous work [25]. Figure $12 \mathrm{~b}$ presents the analysis of the first derivative which in more detail shows the phase transition temperature. As can be seen for scenario no. 1 (transparent ionogel phase), there is only one phase transition with $T_{\mathrm{gs}}=83^{\circ} \mathrm{C}$, for scenario no. 2 (mixed gel phase), two phase transition temperatures were detected, $T_{\mathrm{gs}}=83{ }^{\circ} \mathrm{C}$ and $T_{\mathrm{gs}}=94^{\circ} \mathrm{C}$, and for scenario no. 3 (opaque ionogel phase) again, we observed one phase transition temperature at $T_{g s}=95^{\circ} \mathrm{C}$. Based on the obtained data, we can conclude that the aggregate type (size and shape) from which the gel matrix is composed, determines the thermal properties of the gel phase. By changing the gelation temperature and kinetics of the cooling stage, we can have an influence on the selfassembly process leading to the creation of different gelator aggregates. By slowing down the kinetics of the cooling, we allow the self-assembly process to act longer, giving more 


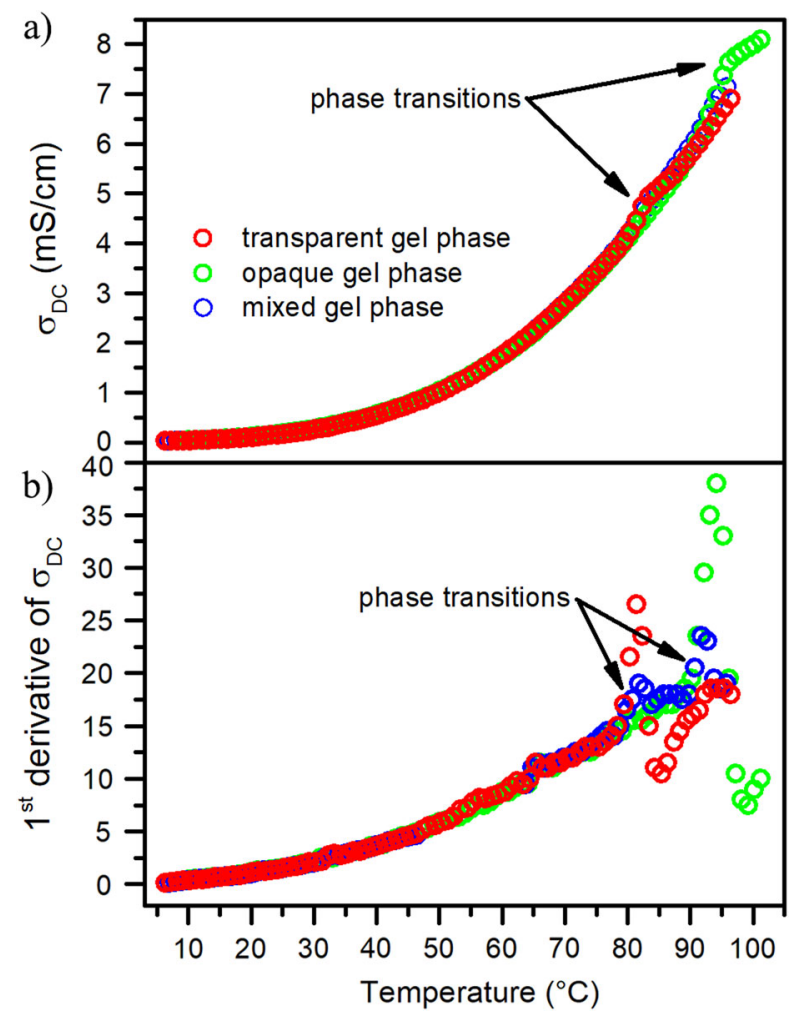

Fig. 12 The heating curve of TSC measurements recorded for $1.0 \mathrm{M}$ TEABr/glycerol ionogel after different scenarios of cooling $\mathbf{a}$, together with analysis of the first derivative $\mathbf{b}$

time to build hydrogen bonds between gelator molecules, what results in building aggregates as much bigger and thicker rods. Because of the much bigger size of gelator aggregates, the gel phase stops to be transparent and starts to scatter the visible light, what results in the opaque state. During fast cooling, there is much less time to build the hydrogen bond network between gelator molecules; thus, created aggregates are much smaller and do not scatter the visible light, so we obtain a transparent gel phase. This means that by only changing the kinetics of the selfassembly process which takes place during gelation, we were able to increase the phase transition temperature by 12 ${ }^{\circ} \mathrm{C}$ in the case of $1.0 \mathrm{M} \mathrm{TEABr} / \mathrm{Glyc}$ electrolyte solution and obtain an ionogel with different optical properties, as illustrated in fig. S9 in supporting information. In the case of $0.5 \mathrm{M} \mathrm{TEABr} / \mathrm{Glyc}$ electrolyte, we have observed the transparent gel phase obtained in scenario no. 1 and the opaque gel phase obtained in scenario no. 3 . However, there was no efficient creation of a mixed gel phase in scenario no. 2 . In the case of a transparent gel phase, we have determined the gel to sol phase transition temperature to be $T_{\mathrm{gs}}=84^{\circ} \mathrm{C}$ (which is very close to what has been observed for a higher concentration of the electrolyte solution), whereas in the case of the opaque gel phase, we were not able to detect the gel to sol phase transition as our TSC setup allows to measure only up to $100^{\circ} \mathrm{C}$. In order to determine where the phase transition temperature is, the sample was subjected to phase transition temperature measurements by visual inspection. According to our previous study about the nature of the anomaly observed in TSC method, we have defined the gel to sol phase transition temperature, the temperature when the bulk flow starts, this happens at the point where $40-60 \%$ of the gel matrix is melted. For the ionogel with $0.5 \mathrm{M} \mathrm{TEABr} / \mathrm{Glyc}$ electrolyte solution in an opaque gel phase, the phase transition temperature was determined to be $T_{\mathrm{gs}}=109^{\circ} \mathrm{C}$. As for the case with $2.0 \mathrm{M} \mathrm{TEABr} / \mathrm{Glyc}$ electrolyte solution, we were able to create only a transparent gel phase and even extending the time for gelation significantly at high temperature (scenario no. 3), we were not able to obtain an opaque ionogel phase as the sample stayed at the sol phase. Based on this observation, we can conclude that the high concentration of ions disturbs the self-assembly process between gelator molecules at high temperatures. However, it still allows obtaining a transparent ionogel sample with rapid cooling. Nevertheless, the gel to sol phase transition temperature in this case was detected at $T_{\mathrm{gs}}=54^{\circ} \mathrm{C}$. The decrease of the phase transition temperature confirms that a high concentration of ions disturbs the self-assembly process based on hydrogen bonding between gelator molecules and leads to weakening of the gel matrix. The TSC curves together with first derivative analysis for an ionogel with 0.5 and 2.0 M TEABr/Glyc electrolyte solution are presented in fig. S10 and fig. S11 in supporting information, respectively.

\subsection{The microstructure of the gelator 3D matrix in the ionogel phase}

The self-assembly process leading to the creation of the 3D gel matrix composed of the gelator molecules is based in current studies on the hydrogen bonding, and thus can be affected by external conditions. By setting different gelation temperatures and temperature change rates (as described in the previous section), we were able to obtain a macroscopically different ionogel phase (fig. S9 in supporting information). Figure 13 shows the microstructure of transparent and opaque ionogel phases displaying differences between them.

As can be seen, both the addition of the salt as well as the different type of thermal treating makes changes in the way the aggregates are built. First, the addition of ions makes that in reference microstructure, which looks like some liquid crystal texture, a new phase is growing which is characterized by much smaller domains. For $0.5 \mathrm{M}$ TEABr/ Glyc electrolyte in the transparent gel phase, we can see domains characteristic of the reference system, resulting in 


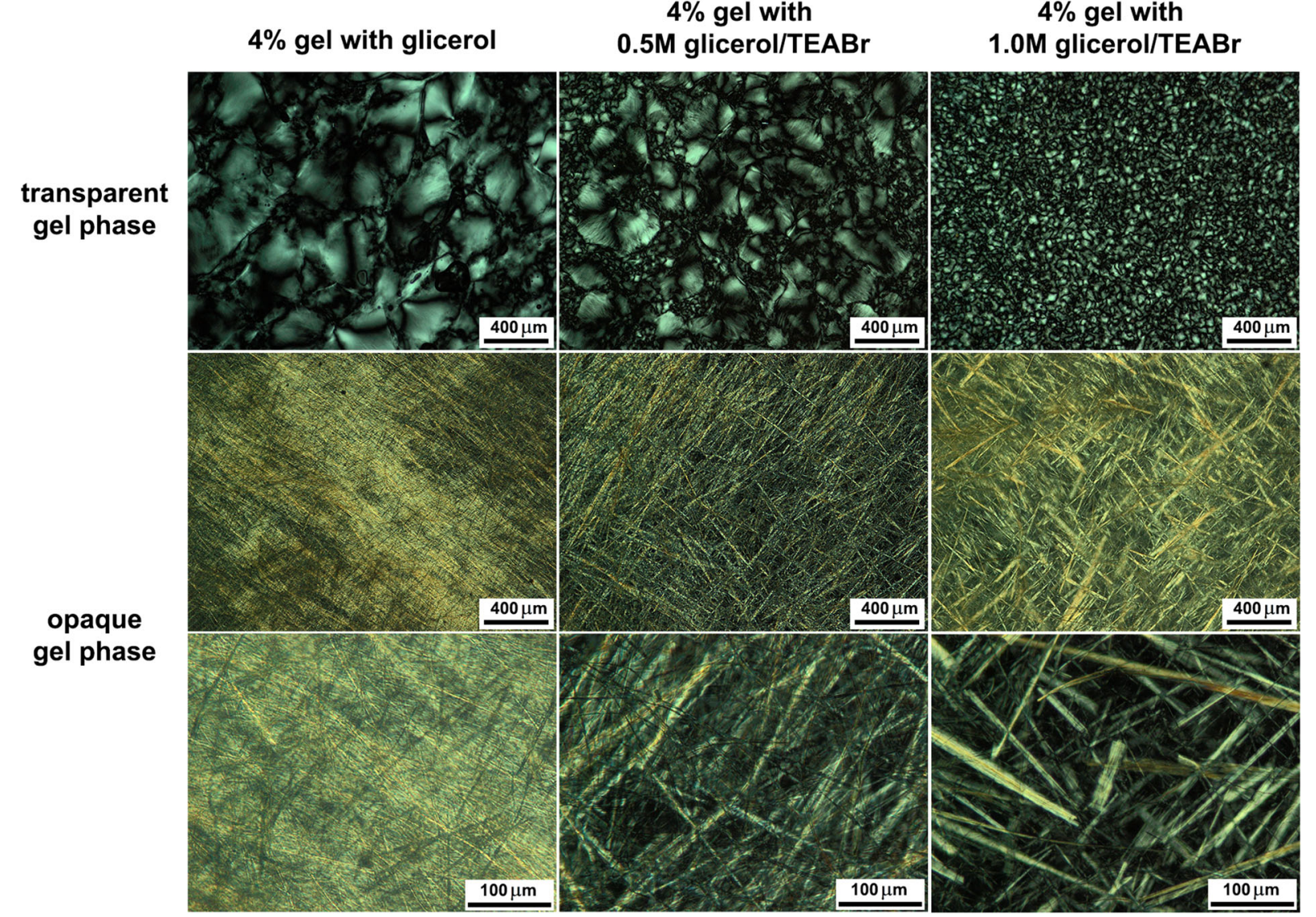

Fig. 13 The microstructure of the reference system (molecular gel with glycerol) and ionogels with TEABr/glycerol electrolyte solutions after different scenarios of the cooling stage

much smaller domains for the ionogel phase. For a $1.0 \mathrm{M}$ concentration of ions, the microstructure is created only from very small domains. Therefore, we can conclude that by adding ions, the size of a single aggregate and thus also a domain created by gelator aggregates is decreasing, what is a consequence of disturbing the hydrogen bond network between gelator molecules by the presence of TEABr ions. By changing the thermal treating of an ionogel during gelation, the form of aggregates is changing significantly from LC like to thin long fibers. Again, addition of salt changes the size of gelator aggregates and makes the fibers shorter and thicker with an increase of the ion concentration. On the basis of the obtained data, we can say that the presence of ions has an influence on the size of a single aggregate and the thermal treating has an influence on the shape of a single aggregate. From our observations, the molecular aggregates in the form of fibers have better thermal properties and higher phase transition temperatures than LC-like textures. Simultaneously, the microstructure (in the sense of size and shape of the aggregates of the gel matrix) does not have an influence on the ionic conductivity properties, showing that LMWG does not disturb the ion mobility. However, the type of the 3D gel matrix determines the temperature range of the ionogel phase existence and can be controlled to obtain either higher phase transition temperatures or desired optical properties of the ionogel phase (transparent or opaque).

\section{Conclusions}

The present work was focused on the study of the external conditions influence on the physical properties of nonaqueous ionogels made with a quaternary ammonium salt and LMWG. The effect of the 3D gel matrix microstructure on the ions' mobility and thermal properties was investigated. It has been shown that the physical gels obtained with lowmolecular-weight gelators form matrices with sufficiently large pores to not restrict the motion of investigated ions. On the other hand, the size and shape of the gelator aggregates can be changed depending on the thermal treating during the gelation stage, what influences directly on the temperature range of the gel phase occurrence and gel to sol phase transition temperature. The NMR spectroscopy studies have shown that the hydrogen bond network between glycerol but also between gelator molecules is 
affected by the presence of the ions. In the case of TEABr, this influence is constructive and leads to a decrease of the static viscosity of the prepared electrolyte solution what positively influences on ionic conductivity. In the case of TBABr, the influence of the ions is negative, leading to an increase of the static viscosity and as a result to a decrease of the conductivity. The NMR diffusometry has shown that some fraction of glycerol creates the solvation shell around the ions and that the solvation shell is preserved at higher temperatures in the ionogel phase than in electrolyte solution. The viscometry technique was successfully used to determine the stability of electrolyte solutions and interactions of ions with glycerol determining the best composition of electrolyte for utilization in ionogels. The TSC method has shown that the ionogels based on LMWG can have tunable properties controlled by the kinetics of the selfassembly process taking place during the gelation stage. With the high precision of a TSC protocol, it is possible to obtain in a highly reproducible way a thermally reversible ionogel with different gel microstructures resulting in a wider temperature range of the gel phase or different optical properties (transparent or opaque samples).

By the obtained results, we can conclude that with the aid of the LMWG, it is possible to create ionogels with high conductive properties and a tunable microstructure. The gelation process is fully reversible allowing to renew the gel structure damaged mechanically or just used in time. Renewed systems are characterized with full reproducibility of nominal parameters. In our opinion, ionogels based on LMWG, thanks to the diversity of chemical compounds, the thermal reversibility of the gelation process, high conductivity, easy manufacturing process, functionality features (tunable temperature range or physical occurrence), and recycling ability can constitute a strong alternative solution for commercially available polymer electrolytes.

Acknowledgements The author would like to thank Mr. Łukasz Chojnacki for assistance during TSC measurements.

\section{Compliance with ethical standards}

Conflict of interest The author declares that he has no conflict of interest.

Open Access This article is distributed under the terms of the Creative Commons Attribution 4.0 International License (http://crea tivecommons.org/licenses/by/4.0/), which permits use, duplication, adaptation, distribution, and reproduction in any medium or format, as long as you give appropriate credit to the original author(s) and the source, provide a link to the Creative Commons license, and indicate if changes were made.

\section{References}

1. Ishioka Y, Minakuchi N, Mizuhata M, Maruyama T (2014) Soft Matter 10:965

2. Hanabusa K, Fukui H, Suzuki M, Shirai H (2005) Langmuir 21:10383

3. Yan J, Liu J, Jing P, Ch Xu, Wu J, Gao D, Fanf Y (2012) Soft Matter 8:11697

4. Minakuchi N, Hoe K, Yamaki D, Ten-no S, Nakashima K, Goto M, Mizuhata M, Maruyama T (2012) Langmuir 28:9259

5. Bideau J, Viau L, Vioux A (2011) Chem Soc Rev 40:907

6. Maršavelski A, Smrečki V, Vianello R, Žinić M, Moguš-Milankovic A, Štanić A (2015) Chem Eur J 21:12121-12128

7. Ikeda A, Sonoda K, Ayabe M, Tamaru S, Nakashima T, Kimizuka N, Shinkai S (2001) Chem Lett 30:1154-1155

8. Bielejewski M, Łapiński A, Demchuk O (2017) J Coll Interface Sci 490:279

9. Atsbeha T, Bussotti L, Cicchi S, Foggi P, Ghini G, Lascialfari L, Marcelli A (2011) J Mol Struct 993:459

10. Gronwald O, Snip E, Shinkai S (2002) Curr Opin Coll Interface Sci 7:148

11. Vintiloiu A, Leroux J-Ch (2008) Control Rel 125:179

12. Wang Z, Fujisawa S, Suzuki M, Hanabusa K (2016) Macromol Symp 364:38

13. Huo ZP, Dai SY, Zhang CG, Kong FT, Fang XQ, Guo L, Liu WQ, Hu LH, Pan X, Wang KJ (2008) J Phys Chem B 112:12927

14. Mohmeyer N, Kuang D, Wang P, Schmidt H-W, Zakeeruddin SM, Grätzel M (2006) J Mater Chem 16:2978

15. Decoppet J-D, Moehi T, Babkair SS, Alzubaydi RA, Ansari AA, Habib SS, Zakeeruddin SM, Schmidt H-W, Grätzel M (2014) J Mater Chem A 2:15972

16. Mohmeyer N, Wang P, Schmidt H-W, Zakeeruddin SM, Grätzel M (2004) J Mater Chem 14:1905

17. Tao L, Huo Z, Ding Y, Li Y, Dai S, Wang L, Zhu J, Pan X, Zhang B, Yau J, Nazeeruddin MK, Grätzel M (2015) J Mater Chem A $3: 2344$

18. Cabeza O, Segade L, Dominguez-Pérez M, Rilo E, GarciaGarabal S, Ausin D, Martinelli A, López-Lago E, Varela LM (2017) J Chem Thermodyn 112:267

19. Kelly T, Ghadi BM, Berg S, Ardebili H (2016) Sci Rep 6:20128

20. Gronwald O, Shinkai S (2001) J Chem Soc, Perkin Trans 2:1933

21. Bielejewski M (2015) Electrochim Acta 174:1141

22. Izumo N, Oda H (2008) J Ceram Soc Jpn: Ceram Jpn 43:200-205

23. Bielejewski M, Nowicka K, Bielejewska N, Tritt-Goc J (2016) J Electrochem Soc 163:G187

24. Kamiguchi K, Kuroki S, Satoh M, Ando I (2009) Macromolecules 42:231

25. Mitra PP, Sen PN, Schwartz LM, Ledoussal P (1992) Phys Rev Lett 68:3555

26. Bielejewski M, Kowalczuk J, Kaszyńska J, Łapiński A, Luboradzki R, Demchuk O, Tritt-Goc J (2013) Soft Matter 9:7501-7514

27. Bielejewski M, Rachocki A, Kaszyńska J, Tritt-Goc J (2018) Phys Chem Chem Phys 20:5803-5817 